\title{
Short-term dexamethasone plus acetylsalicylic acid treatment during in vitro fertilization procedure
}

\author{
Dejan Mitic ${ }^{1}$, Jelena M. Milenkovic'², Maja Milojkovic², \\ Miljan Jeremic ${ }^{3}$, Aleksandra Petric ${ }^{1}$, Marin Basic ${ }^{1}$ \\ ${ }^{1}$ Gynecology and Obstetrics Clinic, Clinical Center Nis, Faculty of Medicine, University of Nis, Serbia \\ ${ }^{2}$ Department of Pathophysiology, Faculty of Medicine, University of Nis, Serbia \\ ${ }^{3}$ Faculty of Medicine, University of Nis, Serbia
}

\begin{abstract}
Objectives: Embryo implantation represents the major limiting step during in vitro fertilization (IVF) procedure. Immunological and coagulation abnormalities were shown to have a substantial part in multifactorial etiology of IVF failure. We aimed to investigate the effect of short-term low-dose dexamethasone plus acetylsalicylic acid (ASA) treatment, starting at the time of embryo transfer, on the implantation and clinical pregnancy rates in general IVF population.

Material and methods: Out of 233 consecutive patients undergoing fresh IVF/intracytoplasmic sperm injection (ICSI) cycles 64 received an adjuvant treatment consisting of dexamethasone $(0.5 \mathrm{mg} /$ day) plus ASA ( $100 \mathrm{mg} / \mathrm{day}$ ) (DA group), starting on the day of embryo transfer. Patients not receiving these medications comprised a control group.

Results: Significantly more patients in DA group had positive $B$-hCG values than controls $(59.38 \%$ vs. $37.67 \%, p=0.004)$ $(\mathrm{OR}=2.42,95 \% \mathrm{Cl}: 1.33-4.41)$. Implantation rate was $26.53 \%$ in DA group and $15.92 \%$ in controls $(p=0.0294)$. Clinical pregnancy rate per started cycle was higher in DA group (43.59\%) than controls $(28.92 \%)$, but the difference was not significant $(p=0.0879 ; \mathrm{OR}=1.99,95 \% \mathrm{Cl}: 0.89-4.41)$.

Conclusions: Our study shows a potential benefit of dexamethasone plus ASA adjuvant treatment in females undergoing IVF/ICSI procedure. As these results show improvement of IVF outcome, a greater number of patients undergoing this type and regime of adjuvant treatment should be investigated.

Key words: embryo implantation; pregnancy rate; assisted reproductive techniques; spontaneous abortion; coagulation disorders; glucocorticoid effects
\end{abstract}

Ginekologia Polska 2019; 90, 4: 201-205

\section{INTRODUCTION}

In vitro fertilization (IVF) procedure is still a challenging process with embryo implantation being the major limiting step for its success. Implantation depends on the quality of embryo and endometrial receptivity. In order to enhance receptivity various adjuvant treatments have been investigated. Immunological factors and maternal thrombophilia were shown to have a substantial part in multifactorial etiology of IVF failure and early miscarriage. In this respect, immunosuppressants and anti-thrombotic agents have been tested [1, 2].

Many studies evaluated the effect of acetylsalicylic acid (ASA) supplementation in females undergoing IVF treatment, however without general conclusion $[2,3]$. It is suggested that low-dose aspirin could increase implantation rates through its anti-thrombotic effect on the newly formed microvasculature, reduced vascular resistance, and ameliorated blood perfusion, as well as an increase in oocyte maturation. Better blood supply of the uterus leads to an increase of endometrial thickness which is prerequisite for implantation $[4,5]$. Also, certain autoantibodies, such as antiphospholipid and anti- $\beta 2$-glycoprotein I antibodies, may predispose to the placental thrombosis in which case anticoagulants are used [6].

Glucocorticoids (GCs) as immunosuppressants and anti-inflammatory agents are proposed to improve implantation by acting on the uterine natural killer (uNK) cells, cytokine expression, and suppression of inflammation [4, 7]. 
They cause down-regulation of pro-inflammatory cytokines while up-regulating anti-inflammatory molecules. Glucocorticoids also inhibit phagocytosis of macrophages and induce apoptosis in distinct subsets of Tlymphocytes, while having less effect on humoral immunity and antibodies production $[8,9]$. Dexamethasone was determined to cause profound and rapid decrease in circulating monocytes and lymphocytes, as well as lymphocyte apoptosis. On the other hand, GCs may also have a positive effect on the immune system, for example, when concurrently acting with other activation-induced signals they support the survival of T lymphocytes $[10,11]$.

However, treatment with GC and ASA medications creates a potential risk for adverse effects (easier development of an infection, endocrine and metabolic disturbances, bleeding problems, etc.) $[3,12]$. There is no general agreement about the dosage, time to start, and the length of the GCs or ASA treatment $[7,13]$. Thus, it is important to determine an effective and safe protocol that would be optimal to improve the outcome of IVF and minimize the chances of side effects.

\section{Objectives}

The aim of our study was to investigate the effect of short-term low-dose dexamethasone plus ASA treatment, starting at the time of embryo transfer (ET), on the implantation and pregnancy rates in general IVF population.

\section{MATERIAL AND METHODS}

\section{Participants and protocol}

Our prospective case-control study included 233 consecutive patients undergoing fresh IVF/ intracytoplasmic sperm injection (ICSI) cycle, at the Gynecology and Obstetrics Clinic, Clinical Center in Nis, Serbia, between September 2017 and October 2018. The principles of the Helsinki Declaration were applied, along with the Medical Faculty Ethics Committee approval (under No. 12-2307-2/15, 10.03.2016).

The participants were allocated to either the group receiving adjuvant dexamethasone plus ASA treatment, called DA group or to a group receiving only standard treatment, as the control group. Inclusion criteria for the DA group were: spontaneous abortion, inherited thrombophilia (heterogenous and homogenous, as well as compound heterozygous polymorphisms in MTHFR (C677T and A1298C) or PAl-1 gene (4G/5G), acquired thrombophilia (anticardiolipin antibodies and lupus anticoagulant), increased coagulation factor I or II levels, decreased antithrombin III, protein C and S levels, anti-nuclear and anti-thyroglobulin antibodies. Patients in the DA group were provided with detailed information regarding dexamethasone plus ASA treatment, as a concomitant therapy during ART procedure at the Clinic.
They decided whether to receive this additional therapy and signed informed consent.

Exclusion criteria was low-molecular weight heparin (LMWH) use for inherited thrombophilia with homozygous, heterozygous or compound heterozygous mutations in coagulation factor V (G1691A — Leiden) and prothrombin G20210A gene, according to appropriate guidelines [14].

Control group comprised patients that did not have thrombophilia or any immunologic condition, thus they were not requiring $\mathrm{LMWH}, \mathrm{ASA}$ or glucocorticoids.

All participants underwent stimulation with "short" or "long" protocol with gonadotropin-releasing hormone $(\mathrm{GnRH})$ agonists or antagonists plus gonadotropins, at appropriate doses. Ovarian response was monitored by serial transvaginal ultrasound, with a Shimadzu ultrasound device, starting on the 6th day of stimulation and two serum E2 level measurements. When two or more follicles reached $18 \mathrm{~mm}$ an injection of $10.000 \mathrm{IU}$ of human chorionic gonadotropin (hCG) was given i.m or s.c., and a transvaginal ultrasound-guided oocytes aspiration was performed 34-36 hours afterwards. Either IVF or ICSI was done according to the clinical indications. Embryo quality was assessed by the day 3 or day 5 embryo grading system, based on the number and size of the cells and the fragmentation rate or by grading the components of the blastocyst. Embryo transfer was done on day 2,3 , or 5 after the aspiration according to the embryo quality. Maximally 3 embryos were transferred into the uterus. Thereafter, all patients were supplemented with progesterone therapy $200 \mathrm{mg} 3$ times a day, vaginally.

Administration of both adjuvant medications started on the day of embryo transfer: dexamethasone in the dose of $0.5 \mathrm{mg}$ per day and ASA $100 \mathrm{mg}$ per day. Dexamethasone treatment lasted until the end of the 12th week of gestation, while the ASA treatment continued until delivery.

Twelve days after ET, the serum B-hCG was determined for biochemical verification of pregnancy (if above $25 \mathrm{mIU} / \mathrm{mL}$ and rising). Clinical pregnancy was assessed 5- 6 weeks after the ET by detection of a gestational sac, normal embryonic echo, and cardiac pulsation, using a transvaginal ultrasound.

Implantation rate was defined as the number of gestational sacs observed divided by the number of embryos transferred. Clinical pregnancy rate represented the percentage of IVF procedures with a favourable outcome, pregnancy per started cycle.

\section{Statistical analysis}

The results were expressed in percentages, means \pm standard deviations (SD) or medians \pm interquartile range (IQR), as required. Pearson's $X^{2}$ test was used to determine whether there is a significant difference between the groups. Fischer exact test was used when the frequency of a category was 
5 or less. The risk was estimated by the odds ratio (OR) with 95\% confidence interval (Cl). According to the Shapiro-Wilk normality test, Student's $t$-test or Mann-Whitney $U$ test was used. Statistical analyses were performed using SPSS 17.0 (SPSS, Chicago, IL, USA) statistical program.

\section{RESULTS}

Out of 233 patients, 64 received adjuvant dexamethasone plus ASA treatment, while 146 patients comprised the control group. Twenty-three patients were excluded due to unperformed embryo transfer. IVF or ICSI procedure was done in $80.7 \%$ and $19.3 \%$ of patients in the DA group, and $82.55 \%$ and $17.45 \%$ in the control group, respectively. No adverse effects of the adjuvant treatment were reported.

Patients' basal characteristics are presented in Table 1. There was no significant difference between the groups concerning age, body mass index, infertility period, infertility etiology, endometrial thickness, mean serum hormone levels, number and quality of embryos.

\begin{tabular}{|c|c|c|c|}
\hline Variables & $\begin{array}{l}\text { DA group } \\
(n=64)\end{array}$ & $\begin{array}{l}\text { Control group } \\
(n=146)\end{array}$ & $\mathbf{p}$ \\
\hline Age [years] & $34.80 \pm 4.06$ & $36.29 \pm 4.67$ & ns \\
\hline BMI [kg/m2] & $23.04 \pm 2.88$ & $24.01 \pm 3.62$ & ns \\
\hline $\begin{array}{l}\text { Duration of infertility } \\
\text { [years] }\end{array}$ & $6.85 \pm 3.88$ & $5.47 \pm 3.25$ & ns \\
\hline Total Gn dose [IU] & $2533.53 \pm 1134.5$ & $1738.79 \pm 729.8$ & $<0.05$ \\
\hline $\begin{array}{l}\text { Endometrial thickness } \\
2 \text { days prior ET [mm] }\end{array}$ & $9.37 \pm 1.46$ & $9.75 \pm 1.78$ & ns \\
\hline Mean serum E2 & $203.5 \pm 157.0$ & $196.4 \pm 155.9$ & ns \\
\hline Mean serum LH & $5.22 \pm 2.07$ & $6.26 \pm 3.20$ & ns \\
\hline Mean serum FSH & $7.95 \pm 3.46$ & $8.00 \pm 3.73$ & ns \\
\hline $\begin{array}{l}\text { Mean transferred } \\
\text { embryo number }\end{array}$ & $2.60 \pm 0.65$ & $2.44 \pm 0.69$ & ns \\
\hline $\begin{array}{l}\text { Mean top quality } \\
\text { embryo number }\end{array}$ & $1.45 \pm 1.25$ & $1.54 \pm 1.31$ & ns \\
\hline $\begin{array}{l}\text { Levothyroxine treated } \\
\text { hypothyreosis }\end{array}$ & $5(10.20 \%)$ & $32(20.38 \%)$ & ns \\
\hline $\begin{array}{l}\text { Bromocriptine treated } \\
\text { hyperprolactinemia }\end{array}$ & $3(6.12 \%)$ & $18(11.46 \%)$ & ns \\
\hline Causes of infertility & n [\%] & n [\%] & \\
\hline $\begin{array}{l}\text { Reduced ovarian } \\
\text { reserve / Ovulatory }\end{array}$ & $6(12.24 \%)$ & $23(14.65 \%)$ & ns \\
\hline Tubal disease & $10(20.41 \%)$ & $28(17.83 \%)$ & ns \\
\hline Idiopathic infertility & $17(34.69 \%)$ & $49(31.21 \%)$ & ns \\
\hline PCOS & $7(14.29 \%)$ & $20(12.74 \%)$ & ns \\
\hline Male related infertility & $18(36.73 \%)$ & $56(35.67 \%)$ & ns \\
\hline $\begin{array}{l}\text { Previous } \\
\text { endometriosis }\end{array}$ & $1(2.04 \%)$ & $7(4.46 \%)$ & ns \\
\hline
\end{tabular}

$\mathrm{BMI}$ - body mass index; DA — dexamethasone plus acetylsalicylic acid; ET - embryo transfer; Gn - Gonadotropins; ns - not significant; PCOS - polycystic ovary syndrome
No significant difference was found in stimulation protocols between the DA and control group. Patients in DA group most frequently underwent $\mathrm{GnRH}$ agonist — long protocol (54.7\%) compared to GnRH agonist - short (10.9\%) and antagonist (34.4\%) protocol, while the protocol frequencies in control group were following: $46.6 \%, 14.4 \%$, and $39.0 \%$, respectively. The doses of gonadotropins were significantly higher in DA group than controls, but this would not influence adjuvant treatment effect on implantation process as this treatment started on the day of ET and there was no difference in the mean number and quality of transferred embryos between the groups.

Significantly more patients in DA group had positive ß-hCG values than controls ( $59.38 \%$ vs. $37.67 \%$; chi $=8.4948$, $\mathrm{p}=0.00356)(\mathrm{OR}=2.42,95 \% \mathrm{Cl}: 1.33-4.41)$.

When patients were analyzed according to the quality of transferred embryos, the difference between DA and control group for those with good quality embryos was significant ( $p=0.0087$ ), but for poor quality embryos, the difference was not significant $(p=0.0993)$.

Implantation rate was $26.53 \%$ in DA group and $15.92 \%$ in controls, which was statistically significant for $p=0.0294$. Clinical pregnancy rate per started cycle was higher in DA group (43.59\%) than in controls (28.92\%), but insignificant ( $p=0.0879, \mathrm{OR}=1.99,95 \% \mathrm{Cl}$ : 0.89-4.41). Twin pregnancies were slightly higher in DA group (15.79\%) than controls (8.43\%) (difference not significant).

\section{DISCUSSION}

Approximately 10 days after fertilization syncytiotrophoblast reaches the capillaries of the endometrium and maternal blood fulfils the lacunae. The implantation process is finished by the second week following fertilization [15]. Inadequate trophoblast invasion may be caused by many factors among which chromosomal anomalies, immune or thrombophilic disorders are most frequent [1]. Thus, it is supposed that immunologic aberrations and altered blood flow may be responsible for many cases of unexplained infertility and recurrent pregnancy losses.

Studies that investigated the effects of GCs, ASA or their combination gained various results concerning ovarian response and IVF success. Most analyses and evidence do not support the routine use of these medications in ART $[3,16]$.

Results on aspirin effects on IVF outcome measures are found both beneficial and insignificant. In most studies, aspirin dose was $100 \mathrm{mg}$ starting along with controlled ovarian hyperstimulation [3]. Two meta-analyses found no beneficial effect of aspirin on clinical pregnancy or live birth rates [2,3]. However, it is also reported that low-dose aspirin improves uterine artery pulsatility index and blood flow velocity, ovarian responsiveness, high-quality embryos rate, implantation and clinical pregnancy rates $[2,4,17]$. 
There is inconsistent evidence of dexamethasone and aspirin safety in IVF patients. In a meta-analysis of 13 trials, there was no significant difference in miscarriage or vaginal bleeding between groups that used aspirin or placebo [3]. In our study, there were no reported side effects of this treatment. Low-dose aspirin (app. $100 \mathrm{mg} /$ day) seems to provide optimal platelets selective effect without changing the bleeding time [18]. Aspirin shifts local production of thromboxane $\mathrm{A} 2$ towards prostacyclin 12 creating vasodilatation which is necessary for the embryo implantation [17].

Prednisone is a proposed treatment for immunologic dysregulation in couples with recurrent miscarriage. Also, GCs were found beneficial in a group of patients with autoantibodies undergoing IVF, while their use in general IVF population showed ambiguous action $[1,19]$. In a couple of studies, GCs increased clinical pregnancy rate without changing live birth rate [7,19-21], whilst in the study of Liu et al. [22] there was no difference in clinical pregnancy rate but with the significantly higher cumulative live-birth rate.

Prednisolone treatment was shown to reduce the number of uNK cells when taken daily from the first day of the menstrual cycle [23]. Uterine NK cells make the highest per cent of leukocytes in the endometrium, with an increasing number during the first trimester. Some studies observed an increased number of uNK in patients with recurrent miscarriage and recurrent implantation failure [23-25]. However, their prognostic value of pregnancy outcome is uncertain. Their phenotype and function rather than numbers determine the local immune response. Normally uNK are non-cytotoxic and involved in angiogenesis, trophoblast recognition, and immunotolerance [26].

Dexamethasone effect on NK cell response depends on the local cytokine milieu. It was shown to enhance the proliferation, reactivity, and survival of primary NK cells, that were stimulated with IL-2 plus IL-12; while, dexamethasone slightly induced primary NK cell death when incubated with IL-15 [27]. Uterine NK cells number was significantly correlated with the IL-15/actin and IL-18/IL-18BP ratios, where the later was associated with an excess of $\mathrm{UNK}$ cells, determined in patients with implantation failure [24]. Also, it seems that different GCs regulate NK cell responses differently, and their action depends on the activation state of lymphocytes as well as the nature of additional stimulants present [11, 27].

Unlike our study, most studies examined the GC action in IVF cycles starting from the day 1 of the downregulation, thus they could assess dexamethasone sensitization of ovaries to gonadotropins, which was not applicable here $[19,20]$. Similar to our study, Duvan et al. [5] used low-dose aspirin (100 mg/day) along with prednisolone (10mg/day) starting on the ET day $(n=56)$, but they showed no significant differences in implantation and pregnancy rates compared to controls. On the contrary, Zhang et al. [25] reported the beneficial effect of intrauterine perfusion of dexamethasone on clinical pregnancy, for patients with recurrent implantation failure and a high number of uNK cells.

Prednisolone plus ASA treatment was reported to improve IVF outcome in women with autoimmune conditions $[28,29]$. Ten $\mathrm{mg}$ of prednisolone plus $100 \mathrm{mg}$ of aspirin for three months before IVF were useful for antinuclear antibodies positive patients [13]. Also, anti-thyroid antibodies (ATA) are determined in $10.5 \%$ in euthyroid infertile women and $13.9 \%$ those with idiopathic infertility $[28,30]$. IVF patients treated with levothyroxine, prednisolone, and ASA had significantly higher pregnancy and implantation rates than untreated or only levothyroxine treated ATA positive patients [28, 31]. Doses of prednisolone or dexamethasone that had been used as an adjuvant IVF therapy are not sufficient to cause reduction of autoantibody titres [13]. However, there is no proof that antibodies can directly harm pregnancy in human but rather be a sign of a dysregulated immune response [1].

Although there was a significantly higher implantation rate and per cent of clinical pregnancies in DA patients than controls, in our study, the pregnancy rate was not statistically significant. The prescribed doses of adjuvant medications seem not to interfere with normal endometrial recognition of embryo quality and the selection process governed by the migration of endometrial stromal cells $[1,32]$. Implantation frequencies of poor quality embryos did not differ significantly between the groups, implying that dexamethasone plus ASA did not ease implantation of low-viable embryos, which would be potentially harmful.

\section{CONCLUSIONS}

Our study shows a potential benefit of the dexamethasone plus ASA adjuvant treatment in females undergoing fresh IVF/ICSI procedure. Implantation rate was significantly increased in DA group than controls, however, the clinical pregnancy rate was on the border of statistical significance. As these results show improvement of IVF success, a greater number of patients undergoing this type and regimen of adjuvant treatment should be investigated.

\section{Acknowledgements}

The work is supported by Project No. III 41018 of the Ministry of Education, Science and Technological Development of Republic of Serbia; and the Project No. 3 of the Faculty of Medicine University of Nis Serbia.

\section{REFERENCES}

1. Larsen EC, Christiansen OB, Kolte AM, et al. New insights into mechanisms behind miscarriage. BMC Med. 2013; 11: 154, doi: 10.1186/17417015-11-154, indexed in Pubmed: 23803387.

2. Khairy M, Banerjee $\mathrm{K}, \mathrm{El}$-Toukhy $\mathrm{T}$, et al. Aspirin in women undergoing in vitro fertilization treatment: a systematic review and meta-analysis. Fer- 
til Steril. 2007; 88(4): 822-831, doi: 10.1016/j.fertnstert.2006.12.080, indexed in Pubmed: 17509593.

3. Siristatidis CS, Basios G, Pergialiotis V, et al. Aspirin for in vitro fertilisation. Cochrane Database Syst Rev. 2016; 11: CD004832, doi: 10.1002/14651858.CD004832.pub4, indexed in Pubmed: 27807847.

4. Hasegawa I, Yamanoto Y, Suzuki M, et al. Prednisolone plus low-dose aspirin improves the implantation rate in women with autoimmune conditions who are undergoing in vitro fertilization. Fertility and Sterility. 1998; 70(6): 1044-1048, doi: 10.1016/s0015-0282(98)00343-4.

5. Duvan $\mathrm{Cl}$, Ozmen B, Satiroglu $\mathrm{H}$, et al. Does addition of low-dose aspirin and/or steroid as a standard treatment in nonselected intracytoplasmic sperm injection cycles improve in vitro fertilization success? A randomized, prospective, placebo-controlled study. J Assist Reprod Genet. 2006; 23(1): 15-21, doi: 10.1007/s10815-005-9003-3, indexed in Pubmed: 16518579 .

6. Ruiz-Irastorza G, Crowther M, Branch W, et al. Antiphospholipid syndrome. The Lancet. 2010; 376(9751): 1498-1509, doi: 10.1016/s0140-6736(10)60709-x.

7. Boomsma CM, Eijkemans M, Keay SD, et al. Peri-implantation glucocorticoid administration for assisted reproductive technology cycles. Cochrane Database Syst Rev. . 2012(6): CD005996, doi: 10.1002/14651858.CD005996.pub3.

8. Pelaia G, Vatrella A, Cuda G, et al. Molecular mechanisms of corticosteroid actions in chronic inflammatory airway diseases. Life Sciences. 2003; 72(14): 1549-1561, doi: 10.1016/s0024-3205(02)02446-3.

9. Schimmer BP, Funder JW. ACTH. Adrenal Steroids, and Pharmacology of the Adrenal Cortex. In: Brunton LL, Chabner BA, Knollmann BC. ed. Goodman and Gilman's: The Pharmacological Basis of Therapeutics. 12th ed. McGraw-Hill Companies Inc, New York 2011: 1209-1235.

10. Parrillo JE, Fauci AS. Mechanisms of corticosteroid action on lymphocyte subpopulations. III. Differential effects of dexamethasone administration on subpopulations of effector cells mediating cellular cytotoxicity in man. Clin Exp Immunol. 1978; 31(1): 116-125.

11. Perez SA, Mahaira LG, Demirtzoglou FJ, et al. A potential role for hydrocortisone in the positive regulation of IL-15-activated NK-cell proliferation and survival. Blood. 2005; 106(1): 158-166, doi: 10.1182/blood-2004-08-3232, indexed in Pubmed: 15755904.

12. Akhanoba F, MacDougall J, Mathur R, et al. Severe systemic candidiasis following immunomodulation therapy in in vitro fertilisation-embryo transfer (IVF-ET). BMJ Case Rep. 2014; 2014, doi: 10.1136/bcr-2013203202, indexed in Pubmed: 24686802.

13. Zhu $\mathrm{Q}, \mathrm{Wu} \mathrm{Li}, \mathrm{Xu} \mathrm{Bo}$, et al. A retrospective study on IVF/ICSI outcome in patients with anti-nuclear antibodies: the effects of prednisone plus low-dose aspirin adjuvant treatment. Reprod Biol Endocrinol. 2013; 11: 98, doi: 10.1186/1477-7827-11-98, indexed in Pubmed: 24093222.

14. American College of Obstetricians and Gynecologists Women's Health Care Physicians. ACOG Practice Bulletin No. 138: Inherited thrombophilias in pregnancy. Obstet Gynecol. 2013; 122(3): 706-717, doi: 10.1097/01. AOG.0000433981.36184.4e, indexed in Pubmed: 23963422.

15. Rendi M, Muehlenbachs A, Garcia R, et al. Female Reproductive System. Comparative Anatomy and Histology. 2012: 253-284, doi: 10.1016/b9780-12-381361-9.00017-2.

16. Dentali F, Ageno W, Rezoagli E, et al. Low-dose aspirin for in vitro fertilization or intracytoplasmic sperm injection: a systematic review and a meta-analysis of the literature. JThromb Haemost. 2012; 10(10): 2075-2085, doi: 10.1111/j.1538-7836.2012.04886.x, indexed in Pubmed: 22900525.

17. Rubinstein M. Low-dose aspirin treatment improves ovarian responsiveness, uterine and ovarian blood flow velocity, implantation, and pregnancy rates in patients undergoing in vitro fertilization: a prospective, randomized, double-blind placebo-controlled assay. Fertility and Sterility. 1999; 71(5): 825-829, doi: 10.1016/s0015-0282(99)00088-6.
18. Clarke RJ, Mayo G, Price P, et al. Suppression of thromboxane A2 but not of systemic prostacyclin by controlled-release aspirin. N Engl J Med. 1991; 325(16): 1137-1141, doi: 10.1056/NEJM199110173251605, indexed in Pubmed: 1891022.

19. Boomsma CM, Macklon NS. Does glucocorticoid therapy in the peri-implantation period have an impact on IVF outcomes? Curr Opin Obstet Gynecol. 2008; 20(3): 249-256, doi: 10.1097/GCO.0b013e3282f8aff5, indexed in Pubmed: 18460939.

20. Keay SD, Lenton EA, Cooke ID, et al. Low-dose dexamethasone augments the ovarian response to exogenous gonadotrophins leading to a reduction in cycle cancellation rate in a standard IVF programme. Human Reproduction. 2001; 16(9): 1861-1865, doi: 10.1093/humrep/16.9.1861.

21. Kalampokas T, Pandian Z, Keay SD, et al. Glucocorticoid supplementation during ovarian stimulation for IVF or ICSI. Cochrane Database Syst Rev. 2017; 3: CD004752, doi: 10.1002/14651858.CD004752.pub2, indexed in Pubmed: 28349525.

22. Liu S, Shi L, Wang T, et al. Effect of low-dose dexamethasone on patients with elevated early follicular phase progesterone level and pregnancy outcomes in IVF-ET treatment: A randomized controlled clinical trial. Clin Endocrinol (Oxf). 2018; 89(6): 771-778, doi: 10.1111/cen.13824, indexed in Pubmed: 30051499.

23. Quenby S, Kalumbi C, Bates M, et al. Prednisolone reduces preconceptual endometrial natural killer cells in women with recurrent miscarriage. Fertil Steril. 2005; 84(4): 980-984, doi: 10.1016/j.fertnstert.2005.05.012, indexed in Pubmed: 16213853.

24. Lédée-Bataille $\mathrm{N}$, Bonnet-Chea $\mathrm{K}$, Hosny $\mathrm{G}$, et al. Role of the endometrial tripod interleukin-18, -15 , and -12 in inadequate uterine receptivity in patients with a history of repeated in vitro fertilization-embryo transfer failure. Fertil Steril. 2005; 83(3): 598-605, doi: 10.1016/j.fertnstert.2004.11.021, indexed in Pubmed: 15749487.

25. Zhang T, Huang $C$, Du $Y$, et al. Successful treatment with intrauterine delivery of dexamethasone for repeated implantation failure. Am J Reprod Immunol. 2017; 78(6), doi: 10.1111/aji.12766, indexed in Pubmed: 28921750.

26. Kalkunte SS, Mselle TF, Norris WE, et al. VEGF C facilitates immune tolerance and endovascular activity of human uterine NK cells at the maternal-fetal interface. J Immunol. 2009; 182(7): 4085-4092, doi: 10.4049/jimmunol.0803769.

27. Morgan DJ, Davis DM. Distinct Effects of Dexamethasone on Human Natural Killer Cell Responses Dependent on Cytokines. Front Immunol. 2017; 8: 432, doi: 10.3389/fimmu.2017.00432, indexed in Pubmed: 28450865.

28. Revelli A, Casano S, Piane LD, et al. A retrospective study on IVF outcome in euthyroid patients with anti-thyroid antibodies: effects of levothyroxine, acetyl-salicylic acid and prednisolone adjuvant treatments. Reprod Biol Endocrinol. 2009; 7: 137, doi: 10.1186/1477-7827-7-137, indexed in Pubmed: 19941670.

29. Taniguchi F. Results of prednisolone given to improve the outcome of in vitro fertilization-embryo transfer in women with antinuclear antibodies. J Reprod Med. 2005; 50(6): 383-388.

30. Poppe K, Velkeniers B, Glinoer D. Thyroid disease and female reproduction. Clin Endocrinol (Oxf). 2007; 66(3): 309-321, doi: 10.1111/j.1365-2 265.2007.02752.x, indexed in Pubmed: 17302862.

31. Weghofer A, Himaya E, Kushnir VA, et al. The impact of thyroid function and thyroid autoimmunity on embryo quality in women with low functional ovarian reserve: a case-control study. Reprod Biol Endocrinol. 2015; 13: 43, doi: 10.1186/s12958-015-0041-0, indexed in Pubmed: 25975563.

32. Teklenburg G, Salker M, Molokhia M, et al. Natural selection of human embryos: decidualizing endometrial stromal cells serve as sensors of embryo quality upon implantation. PLoS One. 2010; 5(4): e10258, doi: 10.1371/journal.pone.0010258, indexed in Pubmed: 20422011. 